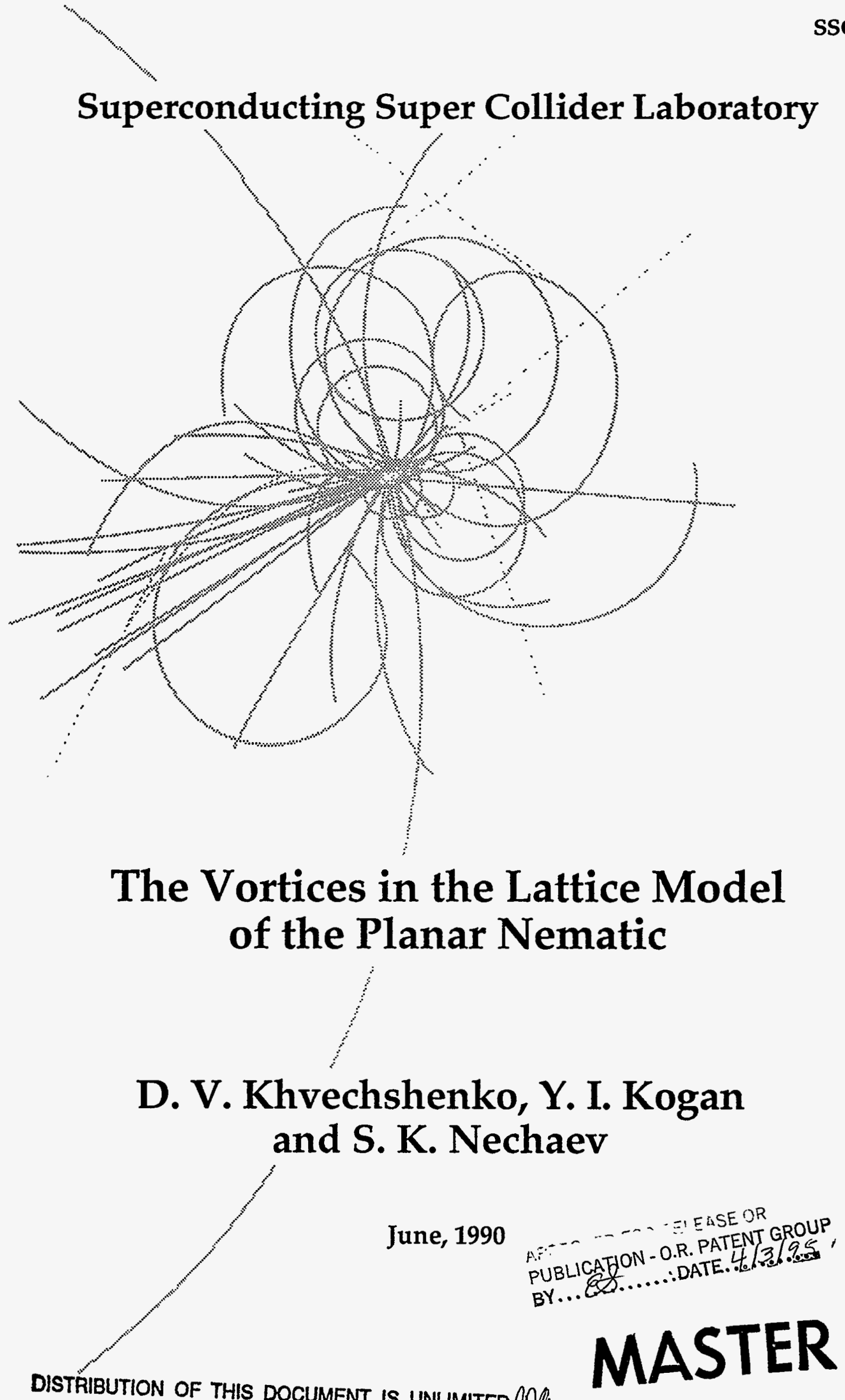


SSCL-282

\title{
THE VORTICES IN THE LATTICE MODEL OF THE PLANAR NEMATIC
}

\author{
D. V. Khvechshenko, ${ }^{*}$ Yan I. Kogan ${ }^{\dagger}{ }^{\text {and S. K. Nechaev }}{ }^{\ddagger}$ \\ Superconducting Super Collider Laboratory $\S$ \\ Physics Research Division \\ 2550 Beckleymeade Avenue
}

Dallas, TX 75237

June 1990

\section{DISCLAIMER}

This report was prepared as an account of work sponsored by an agency of the United States Government. Neither the United States Government nor any agency thereof, nor any of their employees, makes any warranty, express or implied, or assumes any legal liability or responsibility for the accuracy, completeness, or usefulness of any information, apparatus, product, or process disclosed, or represents that its use would not infringe privately owned rights. Reference herein to any specific commercial product, process, or service by trade name, trademark, manufacturer, or otherwise does not necessarily constitute or imply its endorsement, recommendation, or favoring by the United States Government or any agency thereof. The views and opinions of authors expressed herein do not necessarily state or reflect those of the United States Government or any agency thereof.

* Present address: L. D. Landau Institute for Theoretical Physics, GSP-1 117940 Kosygin St., 2, Moscow V-334, USSR

$\dagger$ Present address: ITEP, 117 259, Moscow, B. Cheremushkinskaya, 25, USSR

Institute for Chemical Physics

$\S$ Operated by Universities Research Association, Inc., under contract with the U.S. Department of Energy under Contract No. DE-AC02-89ER40486. 


\section{DISCLAIMER}

Portions of this document may be illegible in electronic image products. Images are produced from the best available original document. 
SSCL-282

\title{
THE VORTICES IN THE LATTICE MODEL OF THE PLANAR NEMATIC
}

\author{
D. V. Khvechshenko, ${ }^{*}$ Yan I. Kogan,${ }^{\dagger}$ and S. K. Nechaev ${ }^{\ddagger}$ \\ Superconducting Super Collider Laboratory $\S$ \\ Physics Research Division \\ 2550 Beckleymeade Avenue \\ Dallas, TX 75237
}

June 1990

\begin{abstract}
The vortices in the planar nematic are considered using the field-theoretical description in terms of the $R P^{2} \sigma$-model. In the strong-coupling expansion the vortices interactions are considered and the new type of phase transition is obtained in the mean-field approximation.
\end{abstract}

* Present address: L. D. Landau Institute for Theoretical Physics, GSP-1 117940 Kosygin St., 2, Moscow V-334, USSR

$\dagger$ Present address: ITEP, 117 259, Moscow, B. Cheremushkinskaya, 25, USSR

$\ddagger$ Institute for Chemical Physics

$\S$ Operated by Universities Research Association, Inc., under contract with the U.S. Department of Energy under Contract No. DE-AC02-89ER40486. 


\section{THE PLANAR RP $P^{2}$ MODEL}

It is natural to describe the planar nematic in terms of the $R P^{2}=S^{2} / Z^{2} 2$-D $\sigma$-model in the same manner as we describe the planar magnetic in terms of the $S^{2}$ or $S^{3}=S U(2) 2$-D $\sigma$-models. The projective sphere $R P^{2}$ appears because the nematic's order parameter-director $\vec{d}$-defines only the line in the $R^{3}$, but not the direction. Thus the effective Hamiltonian is

$$
S=\frac{1}{2 g^{2}} \int \partial_{\mu} \vec{d} \partial_{\mu} \vec{d} d^{2} r=-\frac{1}{2 g^{2}} \sum_{i j} \vec{d}_{i} \vec{d}_{j}+\text { const } \quad \vec{d} \in R P^{2}
$$

where we sum over the nearest neighbor bonds $i j$ in the lattice version. Using the Berezinsky-Villain formalism [1,2] we can rewrite (1.1) as $S^{2} \sigma$-model with auxiliary $Z_{2}$ gauge field:

$$
\begin{aligned}
S & =\frac{1}{2 g^{2}} \sum_{i j}\left(\overrightarrow{n_{i}}-U_{i j} \overrightarrow{n_{j}}\right)^{2} \\
& =-\frac{1}{2 g^{2}} \sum_{i j} \overrightarrow{n_{i}} \overrightarrow{n_{j}} U_{i j}+\text { const }, \overrightarrow{n_{i}} \in S^{2}, U_{i j} \in Z_{2} .
\end{aligned}
$$

The statistical sum takes the form

$$
\begin{aligned}
Z & =\sum_{\{U\}} Z_{\{U\}} \\
Z_{\{U\}} & =\int \mathcal{D} \vec{n} \exp \left[\sum_{i j} \frac{1}{g^{2}} \overrightarrow{n_{i}} \overrightarrow{n_{j}} U_{i j}\right]=\int \mathcal{D} \vec{n} \prod_{i j} e^{\frac{1}{g^{2}} \overrightarrow{n_{i}} \overrightarrow{n_{j}} U_{i j}},
\end{aligned}
$$

where $\{U\}$ is the class of gauge equivalence. Action (we'll use later the fieldtheoretical terminology) (1.2) at $U_{i j}=1$ equals to the $0(3) \sigma$-model lattice action with the continuum limit

$$
S_{0(3)}=\frac{1}{2 g^{2}} \int d^{2} r \partial_{\mu} \vec{n} \partial_{\mu} \vec{n} \quad \vec{n} \in S^{2}
$$

But it is impossible to get the continuum limit of (1.2) in the case of the nontrivial $U_{i j}$ due to the discrete nature of $U_{i j} \in Z_{2}$. The continuum limit 
is described by (1.1). The nontrivial configurations of $U_{i j}$ corresponds to the classical vortices configurations of field $\vec{d}$. These configurations arise because of nontrivial group $\pi_{1}\left(R P^{2}\right)=\pi_{1}\left(S^{2} / Z_{2}\right)=Z_{2}$. Using parameterization $\vec{d}=$ $(\vec{n},-\vec{n}), \vec{n} \in S^{2}$ the vortex solution can be written, for example, as

$$
\begin{aligned}
n_{1} & =\cos \frac{\phi}{2} \\
n_{2} & =\sin \frac{\phi}{2} \\
n_{3} & =0
\end{aligned}
$$

where $\phi$-angle coordinate on the plane. When $\phi$ changes from 0 to $2 \pi, \vec{n}$ changes to $-\vec{n}$ and this is the solution on $R P^{2}$. As usual (see[3]) this solution has logarithmically large action

$$
S=\frac{1}{2 g^{2}} \int r d r d \phi \frac{1}{r^{2}}\left(\partial_{\phi} \vec{n}\right)^{2}=\frac{\pi}{8 g^{2}} \ln \frac{A}{a^{2}},
$$

where $A$ is the total area of the plane, $a$ is the ultraviolet cut-off, the size of the plaquette in the lattice model.

Using the standard argument of the Berezinsky-Kosterlitz-Thouless (BKT) theory $[1,2,3]$ and comparing (1.6) with the vortex entropy one can lead to a conclusion about BKT phase transition at $g^{2}=\pi / 8$, but the story is not so simple. The reason is that $S^{2}$ or $R P^{2} \sigma$-models are asymptotically free [4]. Thus $g$ depends on the scale $\rho$ and in the one-loop approximation

$$
\frac{1}{g^{2}(\rho)}=b \ln \frac{\rho_{c}}{\rho}
$$

where $\rho_{c}$ is the correlation radius which defines the mass scale. Taking into account the quantum corrections the vortex-vortex action (for $Z_{2}$ antivortex and vortex are the same) equals to

$$
S_{1-\mathrm{loop}}=\frac{\pi}{8 g^{2}(\rho)} \ln \frac{\rho^{2}}{a^{2}}=\frac{b \pi}{4} \ln \frac{\rho^{c}}{\rho} \ln \frac{\rho}{a}
$$

and comparing (1.7) with the entropy $\ln \rho^{2} / a^{2}$ we see that at $\rho \sim \rho_{c}$ the last is larger, i.e., the quantum corrections shift the effective charge (or temperature) 
$g(\rho)$ sufficiently large and vortices will be in plasma phase, where the mass gap exists. The most adequate description of this phase is the high-temperature (strong coupling) expansion, which leading approximation is determined by the two first terms in the expansion $I / g^{2}$ :

$$
e^{\frac{1}{g^{2}} \overrightarrow{n_{i}} \vec{n}_{j} U_{i j}} \simeq 1+\frac{1}{g^{2}} \overrightarrow{n_{i}} \overrightarrow{n_{j}} U_{i j}
$$

In this approximation the statistical sum $Z_{\{U\}}$ takes the form:

$$
Z_{\{U\}}=\int \mathcal{D} \vec{n} \prod_{i j}\left(1+\frac{1}{g^{2}} \overrightarrow{n_{i}} \overrightarrow{n_{j}} U_{i j}\right)
$$

where the integration measure $\mathcal{D} \vec{n} \prod_{i} d \overrightarrow{n_{i}}$ is determined in a usual way:

$$
\begin{aligned}
\int d \overrightarrow{n_{i}} \cdot 1 & =1 \\
\int d \overrightarrow{n_{i}} n_{i}^{a} & =0 \\
\int d \overrightarrow{n_{i}} n_{i}^{a} n_{i}^{b} & =\frac{1}{3} \zeta^{a b},
\end{aligned}
$$

etc.

When calculating (1.8) we find that the statistical sum $Z_{\{U\}}$ comes to the sum over the closed contours [5]:

$$
Z_{\{U\}}=\sum_{n=0}^{\infty} 3^{n} \sum_{\left\{c_{1} \ldots c_{n}\right\}}\left(\frac{1}{3 g^{2}}\right)^{L\left(c_{1}\right)+\cdots+L\left(c_{n}\right)} \prod_{i=1}^{n} U\left(c_{i}\right)
$$

where $c_{1}, \ldots, c_{n}$ are the closed contours on the plane, with the self-intersections, generally speaking, and $U(c)=\prod_{i j \in c} U_{i j}$ is the gauge invariant phase factor along the curve, and $c$ is the $Z_{2}$ analog of the Wilson line $\exp \left(i \oint_{c} A_{\mu} d x^{\mu}\right)$. 
We sum in (1.9) on the number of contours $n$ and as usual the natural desire appears for some expression which depends only on one contour. At first sight the sum on $N$ in (1.9) leads to the exponentiation:

$$
Z_{\{U\}}=\exp \left[\sum_{c} 3 e^{-L(c) \ln 3 g^{2}} e^{i \Phi(c,\{U\})}\right]
$$

where $e^{i \Phi(c,\{U\})}=U(c,\{U\})$ is the phase factor for the contour $c$ at given gauge configuration $\{U\}$. However, (1.10) is not the precise analog of (1.9) because of some of the troubles arising in the case of the self-intersecting contours. To avoid these troubles we can either work with the honeycomb lattice, where the self-intersecting contours do not arise due to the odd number of bonds (three) which are going out from one point on a honeycomb lattice, or we can remember that (1.8) is the high-temperature approximation only where the contours with the multiple-passaged bonds are not taken into account. In any way we get (1.10) where the sum spreads on the nonself-intersecting contours only [6].

Thus in the high-temperature or strong coupling limit, the calculation of the some vortices configuration free energy comes to the summation over the closed contours in the "external" field. This field manifests itself as a phase factor $U(c,\{U\})=e^{i \Phi(c,\{U\})}$, where the flux through the contour is

$$
\Phi(c,\{U\})=\pi m
$$

where $m$ is the number of vortices inside $C$. The average flux density, i.e., the flux per plaquette, cannot be larger than $\pi$ evidently (see Section 2). Let us note that in the case of general contours with self-intersections

$$
\Phi(c,\{U\})=\sum_{i=1}^{m} \pi n_{i}
$$

where $n_{i}$ is the winding number of the contour $C$ over the $i$-th vortex. 
The full statistical sum of the $R P^{2}$ model in the strong-coupling limit is

$$
\begin{aligned}
Z_{R P^{2}} & =2^{S} \sum_{\{U\}} Z_{\{U\}}=2^{S} \sum_{\{U\}} e^{-F\{U\}} \\
& =2^{S} \exp \left[3 \sum_{c} e^{-L(C) \ln 3 g^{2}}\right] \sum_{\{U\}} e^{3 \sum_{c} e^{-L(C) \ln 3 g^{2}}[U(c)-1]} \\
& =Z_{S^{2}} \cdot 2^{S} \sum_{\{U\}} \exp \left[3 \sum_{c} e^{-L(C) \ln 3 g^{2}}\right][U(c,\{U\})-1] \\
& =Z_{S^{2}} 2^{S} Z_{v}
\end{aligned}
$$

where the statistical sum of the $0(3) \sigma$-model $Z_{S^{2}}$ is a normalization factor here, $Z_{v}$ is the statistical sum of vortices and equals to the sum of the zero-, one-, two-, and etc. vortices contributions: $Z_{v}=\sum_{m=0}^{\infty} Z^{(m)}$, where

$$
\begin{aligned}
& Z^{(0)}=1 \\
& Z^{(1)}=\int \frac{d^{2} a}{\epsilon^{2}} \exp \left[-6 \sum_{c_{-}(a)} e^{-L\left(c_{-}(a)\right) \ln 3 g^{2}}\right] \\
& Z^{(2)}=\frac{1}{2} \int \frac{d^{2} a}{\epsilon^{2}} \frac{d^{2} b}{\epsilon^{2}} \exp \left[-6 \sum_{c_{-}(a)} e^{-L\left(c_{-}(a, b)\right) \ln 3 g^{2}}\right],
\end{aligned}
$$

here $S=\int d^{2} a / \epsilon^{2}$ is the number of possible vortex's positions on the plane, and in the sum on $c_{-}(a)$ there are only contours which contain point $a$ (with odd winding number in the general case of the self-intersecting curves), $c_{-}(a, b)$ means the odd total winding number for two points $a$ and $b$ and so on. The gauge group volume factor $2^{S}$, which we shall omit later, in the case of generad group $G$ equals to $(\operatorname{dim} G)^{S}$. 


\section{THE PHASE TRANSITION IN THE VORTICES SYSTEM IN THE MEAN FIELD APPROXIMATION}

Consider now the opposite limit when there are as many vortices as plaquettes, i.e., there is flux $\Phi=\pi$ at each plaquette. Using equations (1.10), (1.11) it is easy to see, that in this situation the total number of the length $L$ contours with the odd winding number equals to the number of contours with length $L$ and area $S=a^{2}(2 K+I), k \in Z$ where $a^{2}$ is the plaquette's area.

For the configurations with the smaller number of the vortices the mean field approximation is very natural, when instead of some nonhomogeneous vortices distribution we deal with the homogeneous flux:

$$
\bar{\Phi}=\pi \frac{m}{N}
$$

where $N=S / a$ is the number of plaquettes on the lattice and $m$ is the number of vortices. Thus all information about vortices is coded now only in the one quantity-the number of vortices $m$ or average flux $\bar{\Phi}$.

It is easy to see that $\bar{\Phi}$ and the contour $C$ area $S$ are conjugate to each other and we can write $Z_{v}$ (see (1.10)) in the form of the path integral in the continuum limit $(L=N a=$ const, $N \rightarrow \infty, a \rightarrow 0)$ :

$$
Z_{v} \simeq Z(\bar{\Phi})=\frac{1}{2 \pi} \int d S e^{i \bar{\Phi} S} Z(S)
$$

where

$$
Z(S)=\sum_{\text {even } L} e^{-L \ln 3 g^{2}} Z(S, L),
$$

where $Z(S, L)$ is defined as:

$$
Z(S, L)=\int \cdots \int \mathcal{D} \vec{r} \exp \left\{-\frac{1}{\epsilon} \oint_{c} d s \dot{\vec{r}}^{2}(s)\right.
$$




$$
\begin{aligned}
& \left.-\frac{\tau}{2 a^{2}} \oint_{c} \oint_{c} d s d s^{\prime} \delta\left[\vec{r}(s)-\vec{r}\left(s^{\prime}\right)\right]\right\} \\
& \cdot \delta\left[S-\frac{1}{2} \oint_{c} d s \vec{A}(\vec{r}(s)) \dot{\vec{r}}(s)\right] .
\end{aligned}
$$

The second term in the exponent in (2.3) appears because we must work with nonself-intersecting contours $(\tau>0)$ and the $\delta$-function picked out the contours with the area $S$, where

$$
\begin{aligned}
& S=\frac{1}{2} \oint_{c} d \vec{r} \vec{A}(\vec{r}) ; \\
& \vec{A}=[\vec{\xi}, \vec{r}], \vec{\xi}=(0,0,1)
\end{aligned}
$$

is the Green formula for the area of the curve $C$. Using (2.3)-(2.4) we get the expression for $Z(\bar{\Phi}, L)$

$$
\begin{aligned}
Z(\bar{\Phi}, L)=\int & \cdots \int \mathcal{D} \vec{r} e^{-\frac{1}{\epsilon} \oint d s\left\{\dot{\vec{r}}^{2}(s)+\frac{i}{2} \epsilon \bar{\Phi}[\vec{r}(s), \dot{\vec{r}}(s)]\right\}} \\
& \times e^{-\frac{r}{2 a^{2}} \oint d s \oint d s^{\prime} \delta\left[\vec{r}(s)-\vec{r}\left(s^{\prime}\right)\right]} .
\end{aligned}
$$

It can be shown[7] that problem of the $Z(\bar{\Phi}, L)$ calculation comes to the calculation of the statistical sum of the $n$-component gauge theory $\psi^{4}, \psi=\left(\psi_{1}, \ldots, \psi_{n}\right)$ in the limit $n \rightarrow 0$ :

$$
\begin{aligned}
Z(\bar{\Phi}, L) & =\frac{1}{2 \pi i} \oint_{a-i \infty}^{a+i \infty} e^{-\lambda L} Z(\bar{\Phi}, \lambda) \\
Z(\bar{\Phi}, \lambda) & =\lim _{n \rightarrow 0} \int \cdots \int \prod_{\sigma=1}^{n} \mathcal{D} \psi_{\sigma} \mathcal{D} \psi_{\sigma}^{*} e^{-F(\psi)} \\
F(\psi) & =\int d \vec{r}\left\{\frac{1}{2}|(\nabla-i \bar{\Phi} \vec{A}) \psi|^{2}+\lambda|\psi|^{2}+\tau|\psi|^{4}\right\} .
\end{aligned}
$$


Unfortunately (2.5) and (2.6) cannot be calculated exactly at nonzero $\tau$ and we start from $\tau=0$, thus including the self-intersecting contours in consideration. Then we shall use some scaling arguments to obtain formulae for nonselfintersecting contours only.

Thus at $\tau=0$ the statistical sum (2.5), or more exactly the probability density $P(\bar{\Phi}, L)$ for the paths in the continuum limit, can be calculated exactly [8]:

$$
P_{0}(\bar{\Phi}, L)=\frac{1}{\pi L \epsilon} \frac{\frac{1}{4} L \epsilon \bar{\Phi}}{\operatorname{sh}\left(\frac{1}{4} L \epsilon \bar{\Phi}\right)} .
$$

In the limit $\Phi \rightarrow 0$,

$$
P_{0}(0, L)=\frac{1}{\pi L \epsilon},
$$

which is the correct probability density for the closed contours with the fixed point on the plane and corresponds to the normalization condition of the function $P_{0}(S, L)$ (see $\left.(2.2)\right) \int_{-\infty}^{\infty} d s P_{0}(S, L)=1 / \pi L \epsilon$. Then the statistical sum on the lattice with the coordination number $Z$ equals

$$
Z_{0}(\bar{\Phi}, L)=Z^{L / \epsilon} P_{0}(\bar{\Phi}, L)
$$

In the Appendix we shall demonstrate that calculation of the statistical sum on the lattice comes to the calculation of the Green function of the Hofstadter problem [9].

Using Eqs. (1.1), (2.2), and (2.7) for $F_{0}\{v\}$ on the square lattice with $Z=4$ we get:

$$
\begin{aligned}
F_{0}(v) & \stackrel{\text { def }}{=} F_{0}(\bar{\Phi}) \\
& =\sigma \sum_{\text {even } L} e^{-L \ln 3 g^{2} 4^{L}} \cdot \frac{1}{\pi L \epsilon} \frac{\frac{1}{4} L \epsilon \bar{\Phi}}{\operatorname{sh} \frac{1}{4} L \epsilon \bar{\Phi}} .
\end{aligned}
$$

Remember, that $P_{0}(\bar{\Phi}, L)$ using in (2.8) was obtained for self-intersecting contours. To be more accurate we take into account the first few terms in (2.8) 
explicitly (for $L=4,6$ ) and starting from $L=8$ we substitute $N$ summing on $L$ by integration. We get then

$$
\begin{array}{r}
F_{0}(\bar{\Phi})=\frac{16 \cos \bar{\Phi}}{\left(3 g^{2}\right)^{4}}+\frac{\left(\frac{46656}{25}+432\right) \cos \bar{\Phi}+48 \cos 2 \bar{\Phi}}{\left(3 g^{2}\right)^{6}} \\
+6 \int_{-\infty}^{\infty} d L\left(\frac{4}{3 g^{2}}\right)^{L} \frac{1}{\pi L} \frac{\frac{1}{4} L \bar{\Phi}}{\operatorname{sh} \frac{1}{4} L \bar{\Phi}}+\text { const }
\end{array}
$$

The entropy of the configuration with flux $\bar{\Phi}$ equals to the entropy of system of $m$ particles on lattice with $N$ positions, where $m / N=\bar{\Phi} / \pi$. Thus

$$
-\bar{S}=\frac{\bar{\Phi}}{\pi} \ln \frac{\bar{\Phi}}{\pi}+\left(1-\frac{\bar{\Phi}}{\pi}\right) \ln \left(1-\frac{\bar{\Phi}}{\pi}\right),
$$

$\bar{\Phi}=0$ corresponds to the system without vortices, $\bar{\Phi}=\pi$ corresponds to the system with one vortex for plaquette.

The total free energy $F_{0}^{\Sigma}$ is determined by the (2.9) and (2.10) and the temperature dependence is contained in the coupling constant $g$ (we can put $\left.g^{2} \sim T\right)$.

The function $F_{0}^{\Sigma}(g, \bar{\Phi})$ is described in Fig. 1. It is easy to see that at $g<g_{c}^{0}$ $\left(g_{c}^{0}=1,31\right)$ the phase transition occurs to the state without vortices $(\bar{\Phi}=0)$. The question about the order of this phase transition is still open.

Now we use some simple scaling relations to take into account the fact that we must work with nonself-intersecting contours. Then for $\bar{\Phi}=0$ we have instead of $(2.7 a)$

$$
P(\bar{\Phi}, L)=\frac{1}{\pi R^{2}}
$$

where

$$
R^{2}=L^{2 \nu}
$$

and critical index $\nu=3 / 4$ for the plane[10]. 


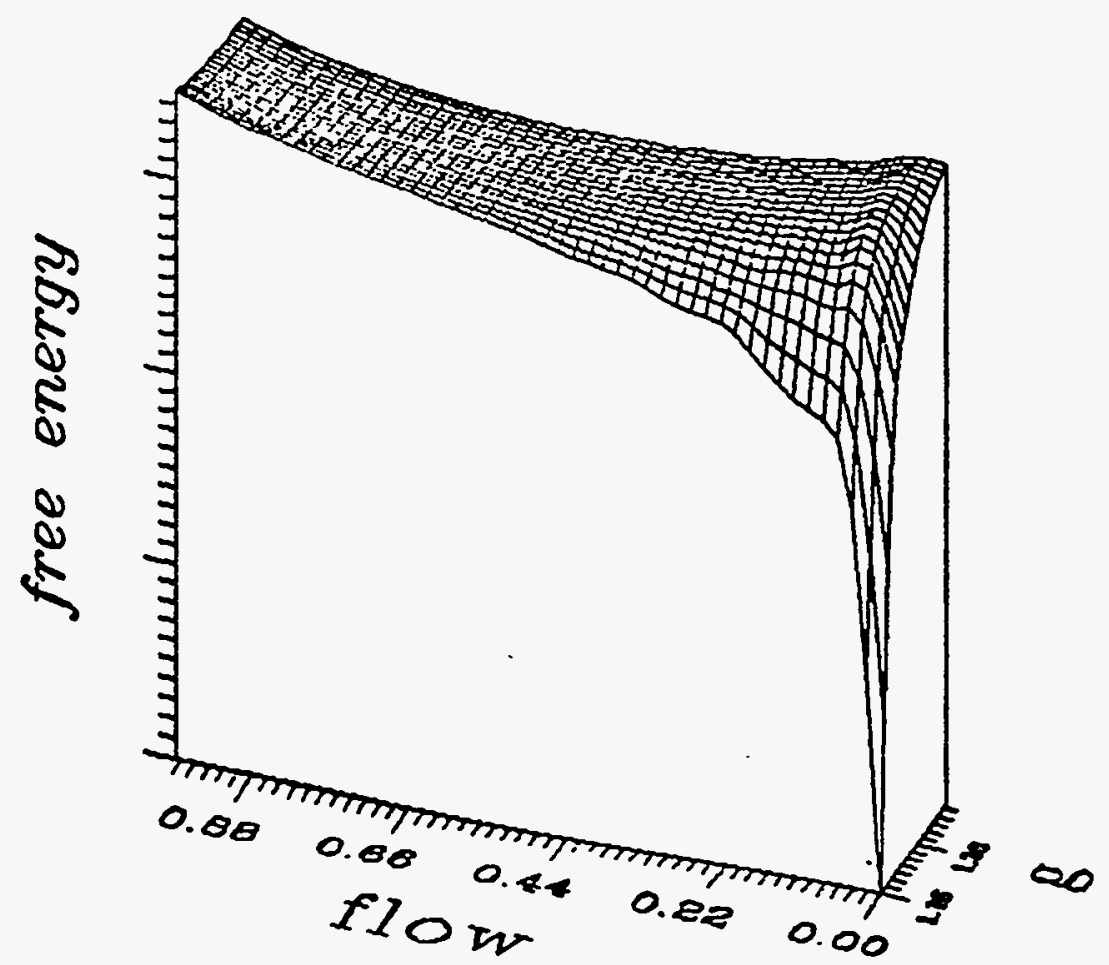

Figure 1.

For $\bar{\Phi} \neq 0$ we can rewrite (2.7) in the form

$$
P(\bar{\Phi}=0, L)=\frac{1}{\pi R^{2}} \frac{\frac{1}{4} R^{2} \bar{\Phi}}{\operatorname{sh}\left[\frac{1}{4} R^{2} \bar{\Phi}\right]}=\frac{1}{\pi L^{2 \nu}} \frac{\frac{1}{4} L^{2 \nu} \bar{\Phi}}{\operatorname{sh} \frac{1}{4} L^{2 \nu} \bar{\Phi}} .
$$

We use the fact that $\bar{\Phi}$ and $S \sim R^{2}$ are the conjugate variables. Using (2.10) and (2.12) we get finally the free energy $F$ :

$$
\begin{aligned}
F^{\Sigma}= & \frac{16 \cos \bar{\Phi}}{\left(3 g^{2}\right)^{4}}+\frac{48 \cos 2 \bar{\Phi}}{\left(3 g^{2}\right)^{6}} \\
& +3 \int_{-\infty}^{\infty} d L\left(\frac{4}{3 g^{2}}\right)^{L} \cdot \frac{1}{\pi L^{2 \nu}} \cdot \frac{\frac{1}{4} L^{2 \nu} \bar{\Phi}}{\operatorname{sh} \frac{1}{4} L^{2 \nu} \bar{\Phi}} \\
& -\frac{\bar{\Phi}}{\pi} \ln \frac{\bar{\Phi}}{\pi}+\left(1-\frac{\bar{\Phi}}{\pi}\right) \ln \left(1-\frac{\bar{\Phi}}{\pi}\right)+\text { const } .
\end{aligned}
$$

The $F^{\Sigma}$ dependence on $g$ and $\bar{\Phi}$ is described on Fig. 2. Now we see that critical value of $g$ is $g=1.21$. 


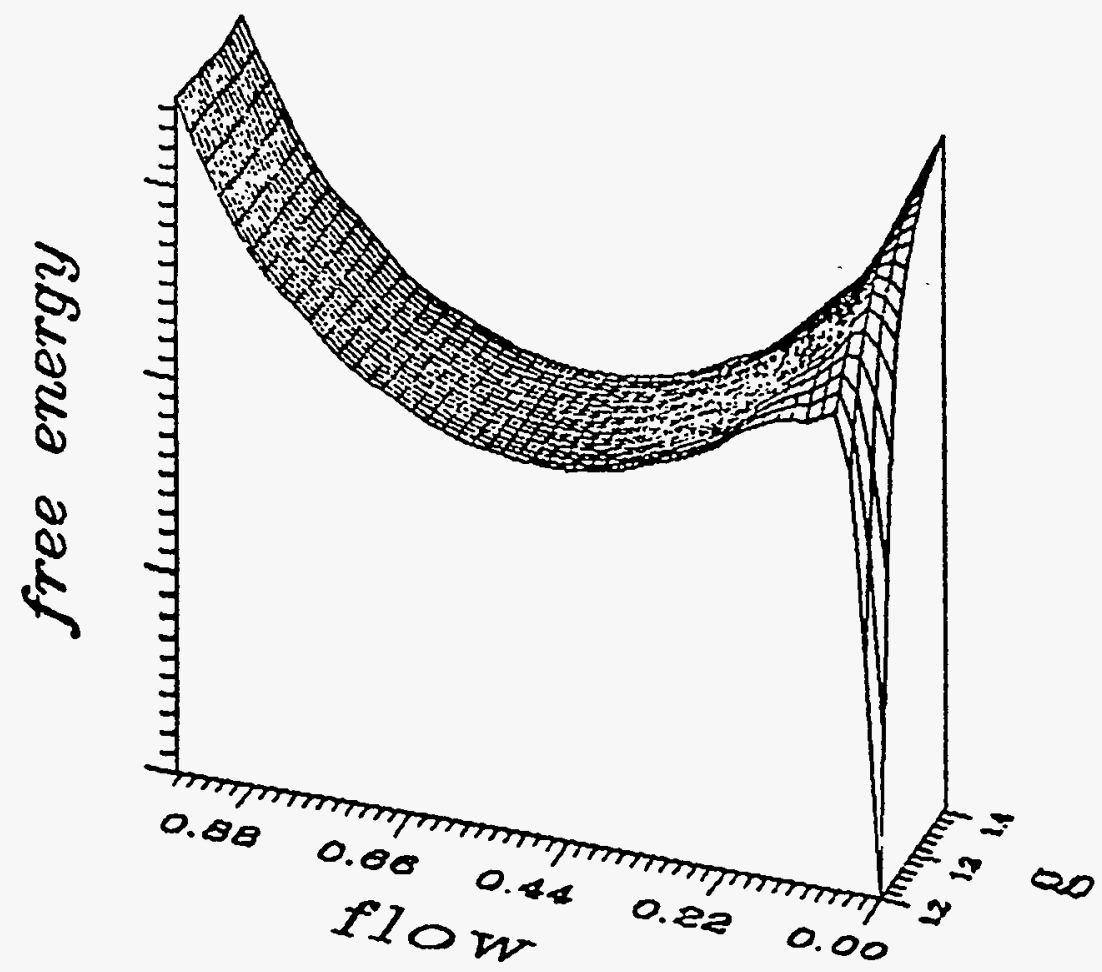

Figure 2.

Thus we obtain the new phase transition in the $R P^{2}$ model (the planar nematic) in the mean field approximation. This picture agrees well with the qualitative arguments concerning the vortex-vortex interaction. Namely, two vortices repulse each other thus there is tendency to diminish the average flux. 


\section{APPENDIX A}

Let us show, that Eq. (2.5) at $\tau=0$ in the discrete version comes to the Hofstadter equation, describing the hopping of 2-D electron in the uniform magnetic field.

The recurrency relation for the statistical sum $Z(\vec{R}, \Phi, L)$ takes the form:

$$
Z(\vec{R}, \bar{\Phi}, L+1)=\int d \vec{R}^{\prime} g\left(\vec{R}-\vec{R}^{\prime}\right) e^{-\frac{i}{2} \bar{\Phi} \vec{\xi}\left[\vec{R}, \vec{R}^{\prime}\right]} Z\left(\vec{R}^{\prime}, \bar{\Phi}, L\right),
$$

where $g\left(\vec{R}, \vec{R}^{\prime}\right)$ is the probability of the one step transition. Taking into account that in the discrete version of the Eq. (A.1) the simple change is needed:

$$
\int d \vec{R}^{\prime} g\left(\vec{R}-\vec{R}^{\prime}\right)(\ldots) \rightarrow \sum W\left(\vec{R}-\vec{R}^{\prime}\right)(\ldots)
$$

where $W\left(\vec{R}-\vec{R}^{\prime}\right)$ is the local hopping matrix, we get the Hofstadter equation:

$$
\begin{aligned}
Z(x, y, \bar{\Phi}, L+1)=\frac{1}{4} & \left\{e^{\frac{1}{2} i \lambda x} Z(x, y-1, \bar{\Phi}, L)\right. \\
& +e^{-\frac{i}{2} \lambda x} Z(x, y+1, \bar{\Phi}, L) \\
& +e^{-\frac{i}{2} \lambda y} Z(x-1, y, \bar{\Phi}, L) \\
& \left.+e^{\frac{i}{2} \lambda y} Z(x+1, y, \bar{\Phi}, L)\right\}
\end{aligned}
$$

where $\bar{\Phi}$ is the flux and (A.3) is written in the gauge $\vec{A}=1 / 2(-H y, H z), H a^{2}=$ $\bar{\Phi}$, here $a^{2}=1$.

Eq. (A.3) cannot be solved exactly for all $\bar{\Phi}$. The solutions are known only for the rational $\bar{\Phi} / \pi$, which is reason for the appearing of the well-known "Hofstadter butterfly" [9]. Here we write as an example the number of the closed contours on the lattice at $\bar{\Phi}=0$ and $\bar{\Phi}=\pi$ :

$$
\begin{aligned}
& Z(\bar{\Phi}=0,2 L)=\left(C_{2 L}^{L}\right)^{2} \\
& Z(\bar{\Phi}=0,2 L)=\sum_{m=0}^{L} C_{L}^{m} C_{2 m}^{m} C_{2 L-2 m}^{L-m} .
\end{aligned}
$$




\section{REFERENCES}

1. V. L. Berezinsky, JETP 59 (C907); 61 (C1144) (1970).

2. J. Villain, J. de Phys. 36, 581 (1975).

3. J. M. Kosterlitz and D. J. Thouless, J. Phys. C 6, 1181 (1973).

4. A. M. Polyakov, Phys. Lett. B (1975).

5. B. Nienhuis, J. Stat. Phys. 34, 731 (1984); in "Phase Transitions and Critical Phenomena," Vol. I (Academic Press, London, 1987); M. den Nijs, Phys. Rev. B 27, 1674 (1983).

6. B. Duplantier, Saclay SPhT/89-162 (preprint).

7. F. Tanaka, Progr. Theor. Phys. 63, 143, 164 (1982).

8. D. C. Khandekar and F. W. Wiegel, J. de Phys. 50, 263 (1989).

9. D. Hofstadter, Phys. Rev. B 14, 2239 (1976); J. Guillement, B. Helffer, and P. Treton, J. de Phys. 50, 2019 (1989).

10. P. De Gen, "Scaling Ideas in Polymer Physics," M (1982). 